\title{
Multiscale light-sheet for rapid imaging of cardiopulmonary system
}

\author{
Yichen Ding, ${ }^{1,2}$ Jianguo Ma, ${ }^{1,3,4}$ Adam D. Langenbacher, ${ }^{5}$ Kyung In Baek, ${ }^{2}$ Juhyun Lee, ${ }^{2}$ \\ Chih-Chiang Chang, ${ }^{2}$ Jeffrey J. Hsu, ${ }^{1}$ Rajan P. Kulkarni, ${ }^{1}$ John Belperio, ${ }^{1}$ Wei Shi, ${ }^{6}$ Sara Ranjbarvaziri, ${ }^{1}$ \\ Reza Ardehali, ${ }^{1}$ Yin Tintut, ${ }^{1}$ Linda L. Demer, ${ }^{1}$ Jau-Nian Chen, ${ }^{5}$ Peng Fei, ${ }^{1,7}$ René R. Sevag Packard, \\ and Tzung K. Hsiai ${ }^{1,2}$ \\ 'Department of Medicine, David Geffen School of Medicine at UCLA, and 'Department of Bioengineering, UCLA, Los \\ Angeles, California, USA. ${ }^{3}$ School of Instrumentation Science and Opto-electronics Engineering, Beihang University, \\ Beijing, China. ${ }^{4}$ Beijing Advanced Innovation Center for Big Data-Based Precision Medicine, Beijing, China. ${ }^{5}$ Department of \\ Molecular, Cell and Developmental Biology, UCLA, Los Angeles, California, USA. ${ }^{6}$ Developmental Biology and Regenerative \\ Medicine Program, Department of Surgery, Children's Hospital Los Angeles, Keck School of Medicine, University of \\ Southern California, Los Angeles, California, USA. ${ }^{7}$ School of Optical and Electronic Information, Huazhong University of \\ Science and Technology, Wuhan, China.
}

\begin{abstract}
The ability to image tissue morphogenesis in real-time and in 3-dimensions (3-D) remains an optical challenge. The advent of light-sheet fluorescence microscopy (LSFM) has advanced developmental biology and tissue regeneration research. In this review, we introduce a LSFM system in which the illumination lens reshapes a thin light-sheet to rapidly scan across a sample of interest while the detection lens orthogonally collects the imaging data. This multiscale strategy provides deep-tissue penetration, high-spatiotemporal resolution, and minimal photobleaching and phototoxicity, allowing in vivo visualization of a variety of tissues and processes, ranging from developing hearts in live zebrafish embryos to ex vivo interrogation of the microarchitecture of optically cleared neonatal hearts. Here, we highlight multiple applications of LSFM and discuss several studies that have allowed better characterization of developmental and pathological processes in multiple models and tissues. These findings demonstrate the capacity of multiscale light-sheet imaging to uncover cardiovascular developmental and regenerative phenomena.
\end{abstract}

Conflict of interest: The authors have declared that no conflict of interest exists.

Published: August 23, 2018

\section{Reference information:} JCI Insight. 2018;3(16):e121396. https://doi.org/10.1172/jici. insight.121396.

\section{Introduction}

Real-time 3-dimensional (3-D) imaging of tissue development and regeneration remains an optical challenge. Conventional optical microscopes are limited by low tissue penetration and small working distance, which are prohibitive to long-term live imaging that requires rapid data acquisition to minimize photobleaching and phototoxicity to the specimens (1-3). In addition, samples must be mechanically sectioned, thereby distorting intrinsic tissue integrity and subsequently resulting in undersampling after 3-D reconstruction (4). While PET $(5,6), \mu \mathrm{CT}(7,8), \mathrm{MRI}(9,10)$, and bioluminescence imaging $(11,12)$ are capable of capturing 3-D images from live samples, the spatial resolution of these techniques is inadequate to capture organ morphogenesis in small-animal models (13-17). For these reasons, the advent of lightsheet fluorescence microscopy (LSFM) (18-22) has revolutionized multiscale imaging, allowing visualization of samples ranging from live zebrafish embryos $\left(\sim 0.4 \times 0.5 \times 0.6 \mathrm{~mm}^{3}\right)$ to adult mouse hearts $(\sim 8 \times 8$ $\times 10 \mathrm{~mm}^{3}$ ) with high-spatiotemporal resolution and minimal photobleaching and phototoxicity.

Unlike confocal and wide-field microscopy, LSFM has the capacity to localize 4-D (3-D spatial + 1-D time or spectra) cellular phenomena with multiple fluorescence channels (23-28). The theoretical principle of light-sheet imaging was first reported in 1903 (29); however, the experimental application of LSFM was not possible until the introduction of fast-rate charge-coupled devices/complementary metal-oxide-semiconductor (CCD/CMOS) camera for high-speed data acquisition in 2004 (23). Initially, LSFM was developed to image small-model organisms, such as Caenorhabditis elegans $(30,31)$, zebrafish embryos $(32,33)$, and Drosophila (34, 35). Subsequently, LSFM imaging of the entire 3-D mouse hippocampus (36-39) and cochlea (40-43) has been made possible with advancements in optical clearance techniques (Figure 1A).

The unique operation of LSFM resides in the orthogonal optical pathway. The illumination and detec- 
tion pathways are linearly aligned in the inverted or upright microscope, whereas the illumination pathway is perpendicular to the detection pathway in the LSFM system. The sample is illuminated at the focal plane of a thin light-sheet of the detection lens (Figure 1, B and C). The emitted fluorescence is perpendicularly collected by the detecting objective lens connected to a fast-rate CCD/CMOS camera. The sample is placed at the intersection of the illumination and the detection axes. In addition to imaging transparent zebrafish embryos, LSFM offers the ability to visualize opaque specimens, including mouse organ systems, following the optical clearing techniques to render these specimens translucent with matching refractive indices $(44,45)$.

A continuous-wave laser is typically used as the illumination source for LSFM. The detection module is composed of a set of filters and a scientific CMOS for rapid multichannel acquisition. This module is perpendicularly installed to the illumination plane (Figure 1, D and E). The lateral resolution (d) of LSFM is determined by the numerical aperture (NA) of the objective lens and the wavelength of excitation light $(\lambda)$, defined as $d$ is proportional to $\lambda / N A$. For data acquisition, each image is acquired within tens of milliseconds of exposure time. Some of the raw data are further processed to remove stationary noise (46-48). In addition to the static light-sheet generated by a cylindrical lens (49-51), the digitally scanned light-sheet microscopy introduced rapid scanning with a Gaussian laser beam (32), further implementing with the two-photon excitation for deep and fast live imaging of Drosophila melanogaster embryos (34).

Recently, improvements in both hardware and software components have enhanced the LSFM-acquired images. First, additional structural illumination or pivoting the light-sheet allows rejection of out-of-focus background and shadows in dense tissues $(52,53)$. Second, computational processing methods have allowed for fusion of multiview images of the same sample $(54,55)$. Finally, four-lens systems have been developed to minimize rotation and registration efforts $(35,56,57)$. Detailed advantages and applications among different light-sheet techniques are listed in Table 1. Distinct from the conventional fluorescence microscopy, LSFM has the capacity to achieve (a) deep penetration into light-scattering tissues; (b) selective optical sectioning of the tissue; (c) minimal photobleaching and phototoxicity; and (d) rapid and multiview acquisition.

In previous work, our group demonstrated the capacity of light-sheet imaging to uncover both mechanical and structural cardiac phenotypes at the cellular level without stitching image columns or pivoting the illumination beams $(18-22,28,58,59)$. In this review, we summarize the use of LSFM to (a) track a recently discovered subpopulation of neural crest-derived cells as they incorporate into the developing heart tube via specific labeling of cell lineages using the fluorescent reporters in zebrafish embryos, (b) capture the dynamics of atrioventricular (AV) valve leaflets throughout the cardiac cycle, (c) integrate dual-sided illumination into the LSFM system to allow for large-scale imaging of 3-D vascular calcification in an adult mouse model, (d) reveal the 3-D developmental lung mesenchyme in a young mouse model, and (e) image the ocular architecture and its retinal vasculature. Overall, the multiscale LSFM system has been shown to be useful in unraveling cardiovascular development and regeneration in models ranging from zebrafish embryos to adult mouse cardiovascular tissues that otherwise have been considered optically challenging for existing imaging modalities.

\section{Time-lapse imaging of neural crest cell incorporation into the developing heart tube}

Genetic lineage-tracing studies have previously shown that cardiac neural crest cells integrate into the developing heart tube and differentiate into cardiomyocytes (60-63). The transgenic NC:NfsB-mCherry zebrafish line (Tg[-5sox10:GAL4,UAS:Cre] la2326Tg; Tg[UAS-E1b:NTR-mCherry] $]^{c 264 T g} ; \mathrm{Tg}[m y l 7: N L S-E G F P]^{c h b 2 T g}$ ) was used for time-lapse imaging to understand the temporal dynamics of how this subpopulation of neural crest-derived cells integrates into the heart tube (Figure 2A). The transgenic $T g\left(N C: N f_{s} B-m C h e r r y\right)$ embryos express nuclear eGFP in cardiomyocytes (green) and mCherry in cells derived from the neural crest lineage (red) (Figure 2B). A series of time-lapse images taken from 26 hours after fertilization (hpf) to $30 \mathrm{hpf}$ revealed the spatial and temporal migration of this subpopulation to the heart tube (Figures 2 , C-G). Neural crest-derived cells were observed contacting the dorsal surface of the heart tube at 26-27 hpf (Figure 2, C and D). By 29 hpf, these neural crest-derived cells integrated into the heart tube and expressed nuclear eGFP (yellow cells in Figure 2, F and G). Thus, LSFM revealed the 4-D distribution of this subpopulation of neural crest-derived cells, providing an imaging platform for further investigation into the lineage-specific differentiation of these cells into cardiomyocytes (yellow in the right panel) and the future septal and valve mesenchyme. 
A

\begin{tabular}{|c|c|c|c|}
\hline Animal model & Sample preparation & Fundamental procedures & Fundamental procedures \\
\hline \multirow{4}{*}{$\begin{array}{l}\text { Zebrafish } \\
\text { embryos }\end{array}$} & \multirow{2}{*}{$\begin{array}{l}\text { 1. Treating fish with } 0.04 \% \\
\text { tricaine methanesulfonate }\end{array}$} & 1. 4-D light-sheet imaging & \\
\hline & & 2. Time-lapse imaging & \\
\hline & \multirow{2}{*}{$\begin{array}{l}\text { 2. Mounting fish in } 0.5 \% \\
\text { low melt agarose }\end{array}$} & 3. Volumetric reconstruction & \\
\hline & & 4. Data computation & \\
\hline \multirow{4}{*}{ Rodents } & 1. Hydrogel embedding & 1. 3-D light-sheet imaging & \multirow{4}{*}{$\begin{array}{l}\text { Optical } \\
\text { clearing }\end{array}$} \\
\hline & 2. Removing lipid & 2. Volumetric reconstruction & \\
\hline & \multirow{2}{*}{$\begin{array}{l}\text { 3. Matching refractive index } \\
\text { (Histodenz) }\end{array}$} & 3. Data computation & \\
\hline & & 4. Data analysis & \\
\hline
\end{tabular}

B

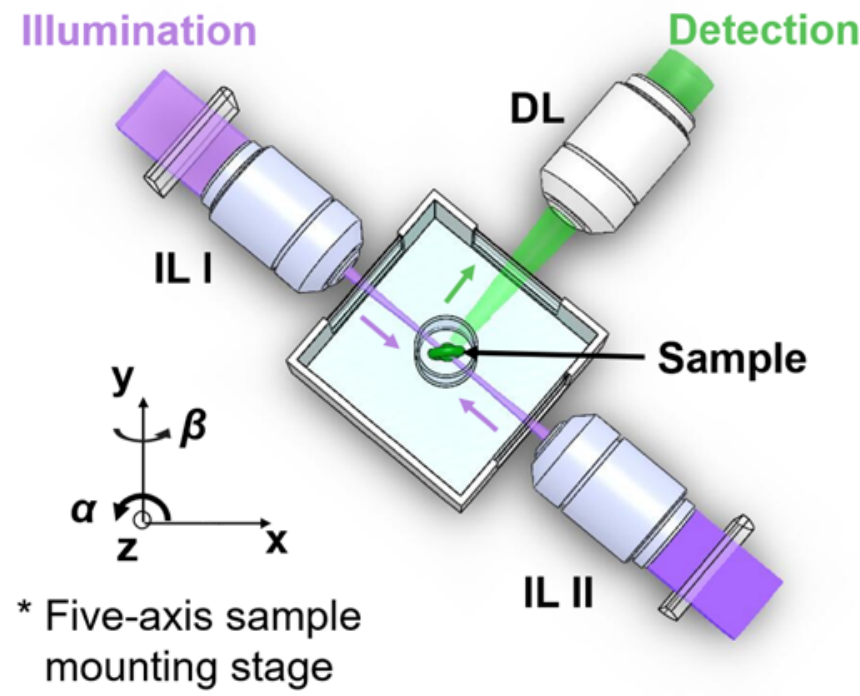

C Laser illumination

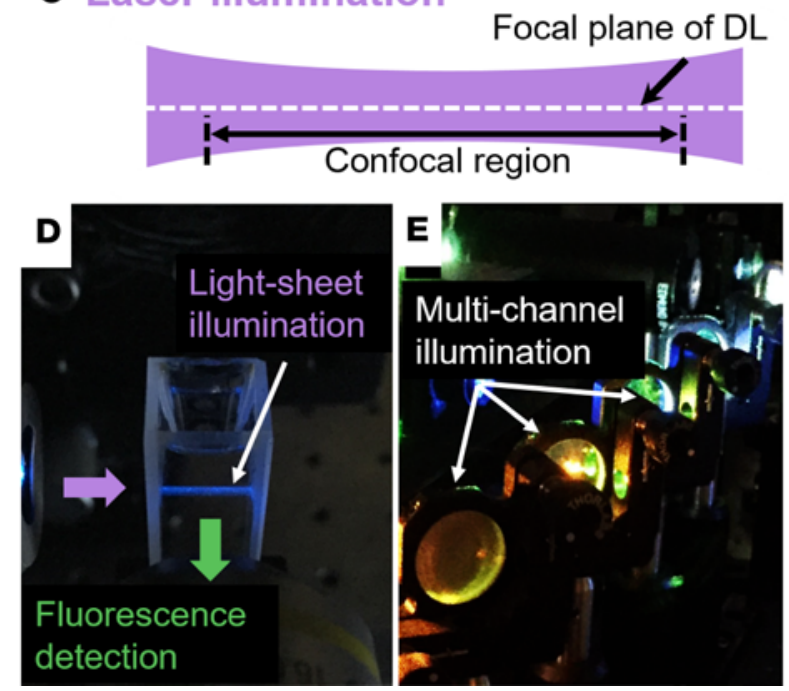

Figure 1. Fundamental concept of the light-sheet imaging strategy. (A) Crucial procedures of multiscale imaging are indicated from embryonic zebrafish and rodent models. (B) The sample holder is oriented by a five-axis mounting stage for scanning the biological specimen. The laser light-sheet is excited from the illumination lenses (IL I and IL II) in a 2-D plane, which is orthogonal to the detection lens (DL). (C and D) A schematic and a photo illustrate the conversion of laser light to a sheet that can transversely illuminate a thin layer of the sample. (E) This photo depicts an array of laser beams aligned for multichannel fluorescent detection. (Reproduced with permission from ref. 68.)

\section{Dual-channel imaging for 3-D valve leaflet dynamics}

Hemodynamic forces are known to govern AV valve formation (64); however, rigorous in vivo visualization of AV leaflets remains an imaging challenge $(65,66)$. Using the transgenic $T g(f l i 1: G F P ; c m l c 2: m C h e r r y)$ zebrafish line at 5 days after fertilization (dpf), we applied dual-channel imaging to capture the excursion of valve leaflets (green) in relation to the endocardium (green) and myocardium (red) (Figure $2 \mathrm{H}$ ). At a rate of 100 frames per second (fps), dual-channel LSFM colocalized AV valve leaflet closure (Figure 2I) and opening (Figure $2 \mathrm{~J}$ ) with ventricular myocardial contraction and relaxation, respectively. These results demonstrate that LSFM imaging allows for time-dependent structure and function determination, which will be useful for studies of mechanosignal transduction of valvulogenesis (67).

In addition to the aforementioned applications of LSFM to examine developmental processes in zebrafish embryos, our group has used LSFM imaging to study vascular injury and regeneration in transgenic $T g(f l i 1: G F P$; gata1:DsRed) and $T g(f l i 1: G F P ;$ cmlc2:mCherry) zebrafish lines, which allowed us to track blood cells in the vasculature in response to tail amputation at $3 \mathrm{dpf}$ (68). In the Tg(fli1:GFP;gata1:DsRed) line, GFP expression is driven by fli1 promoter in the vasculature throughout embryogenesis and the gata1 promoter drives the expression of DsRed in blood cells. These animals allowed us to simultaneously track the vascular loop connection between the dorsal aorta and the dorsal longitudinal anastomotic vessel at 3 $\mathrm{dpf}$ and blood cell trafficking to the injured site. This method may allow studies of nonlinear shear rates in 
Table 1. Overview of different light-sheet techniques

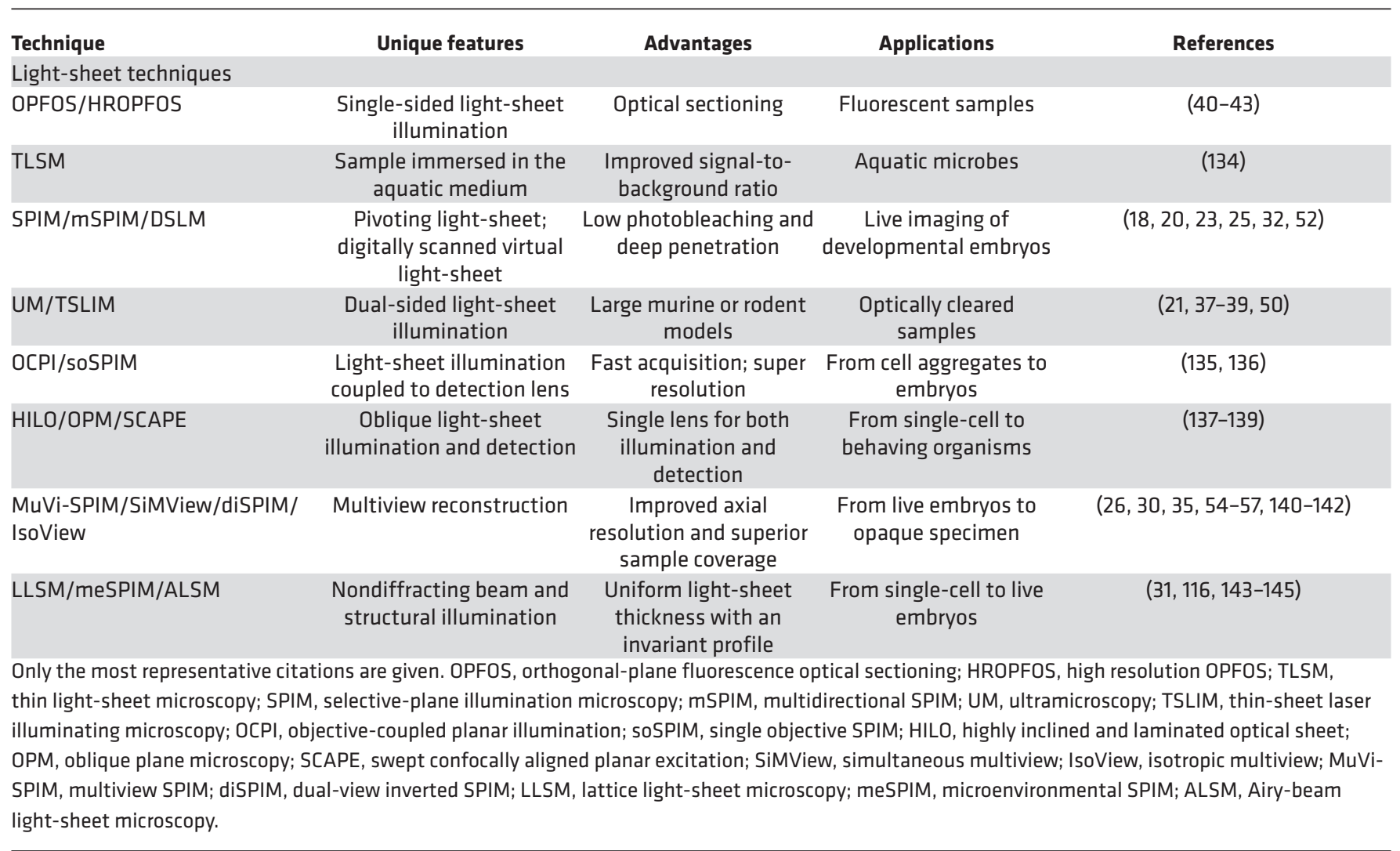

a low-flow Reynolds number system $(\mathrm{Re}=100-1,000)$. Genetic manipulation and use of ADAM10, which inhibits proteolytic cleavage of the Notch extracellular domain, also enabled us to elucidate a mechanism by which Notch-mediated vascular regeneration connects the loop between dorsal longitudinal anastomotic vessel and dorsal aorta. Thus, LSFM provides an entry point for the discovery of novel microcirculation phenomenon with clinical significance for injury and repair.

In summary, light-sheet imaging allows for analysis of the entire 3-D dynamic process of neural crest cell migration to the heart tube and differentiation to cardiomyocytes. Conventional optical microscopes are limited by the insufficient frame rate to 2-D planar images. In studies of cardiomyopathy mechanisms, LSFM has been used to associate neural crest cell-derived cardiomyocytes with trabeculation and cardiac contractility (69-75), with minimal photobleaching and phototoxicity during time-lapse imaging. In addition, this method has been used to quantify the velocity of circulating blood cells (76) in a transgenic zebrafish model of tail injury, allowing for correlation of microcirculatory shear rates with vascular regeneration and providing clinical relevance to the use of selective-plane illumination (77). Due to the cyclical rhythm of myocardial contraction and relaxation, LSFM has marked potential to deliver 4-D reconstruction of dynamic AV valve leaflet movements and, after applying spatial registration between images captured at different time points (20), can provide further insights into the mechanosignal transduction of valvulogenesis.

\section{Analysis of doxorubicin chemotherapy-induced cardiac injury and repair}

The regenerating myocardium electrically couples with uninjured myocardium (78) and represents an evolutionarily conserved model of cardiomyopathy (79) in adult zebrafish. However, the small size of the two-chambered zebrafish heart limits precise morphologic assessment of regenerating heart tissue. Utilization of chemical clearing to achieve tissue transparency and laser light transmission, along with light-sheet imaging coupled with automated image segmentation based on histogram analysis, led to rapid and robust 3-D cardiac reconstruction, thereby unraveling the architecture of doxorubicin chemotherapy-induced cardiac injury and regeneration in adult zebrafish (Figure 2K) (58). Precise 3-D reconstruction further enabled 

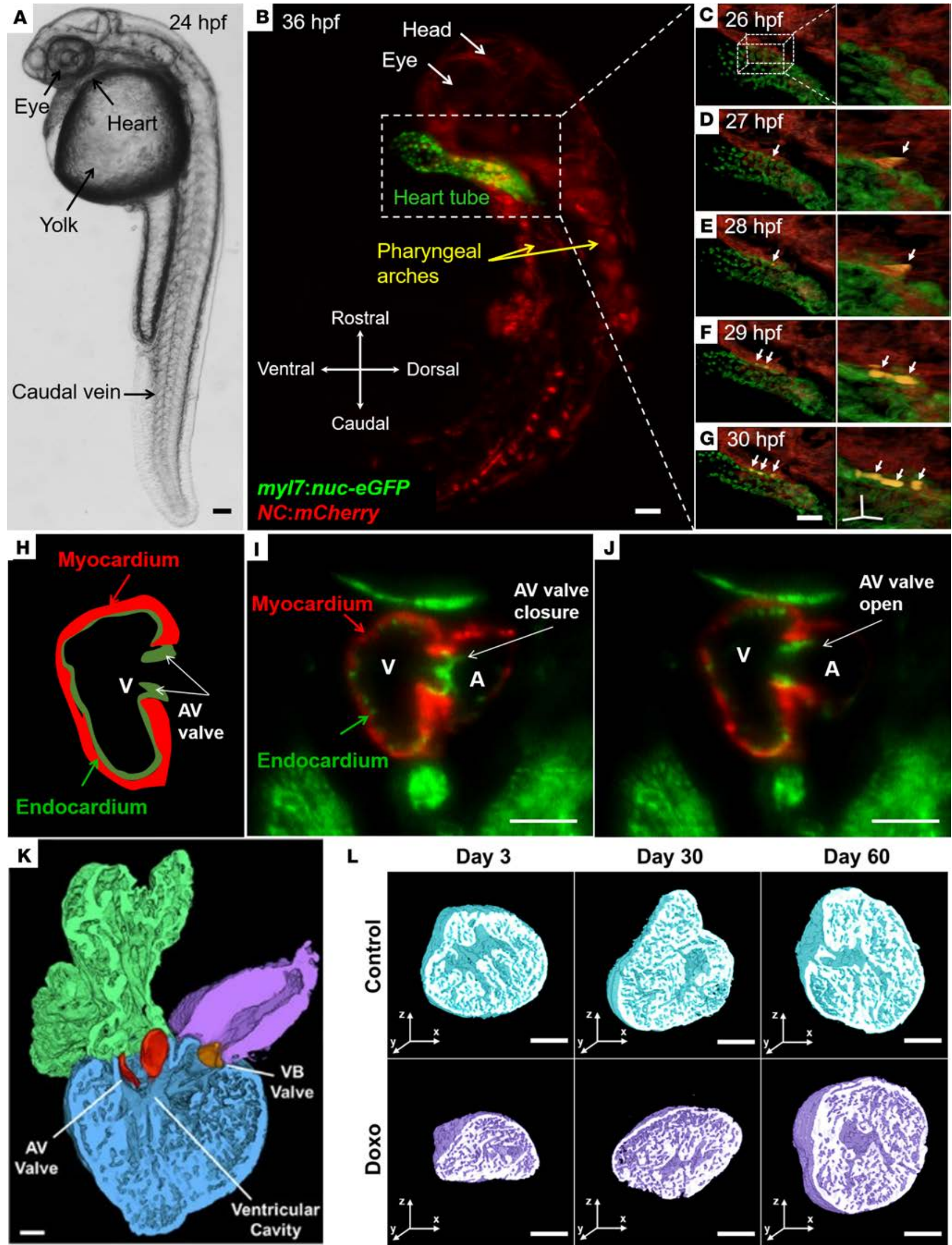

$\mathbf{L}$
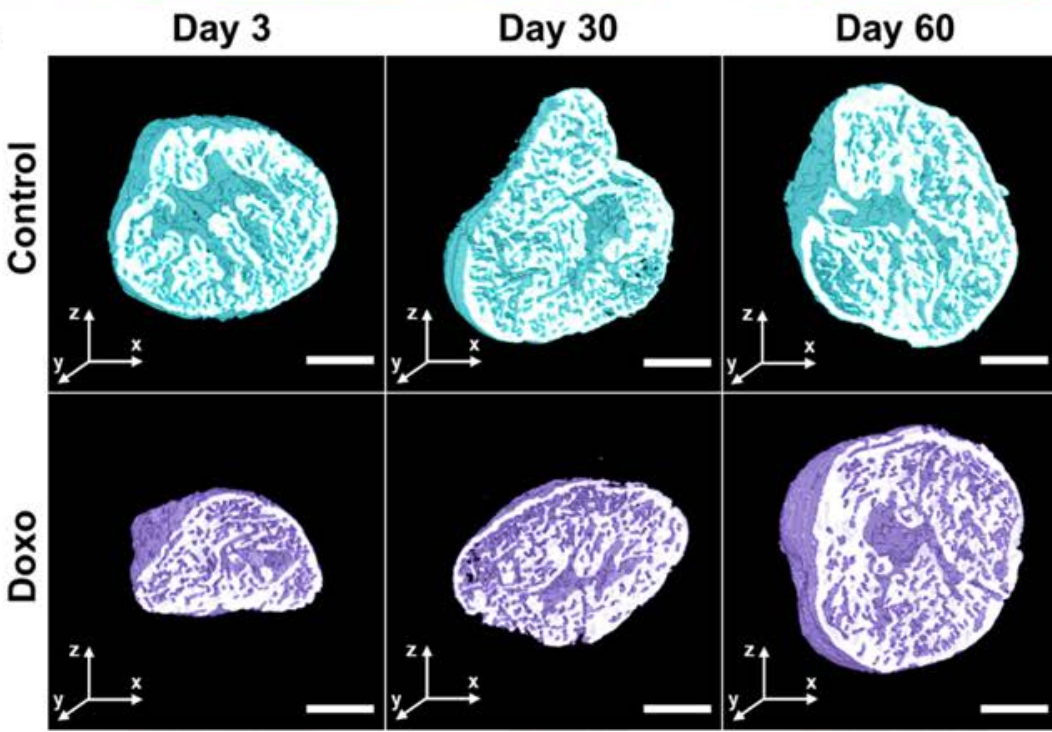
Figure 2. In vivo visualization of the developing hearts from the live zebrafish embryos and high-resolution imaging of chemotherapy-induced cardiac injury and regeneration. (A-G) Light-sheet imaging of neural crest incorporation into the developing zebrafish heart tube (transgenic zebrafish line, Tg(-5sox10:CAL4,UAS:Cre) (1az326Tg; Tg(UAS-E1b:NTR-mCherry) c264Tg; Tg(myl7:NLS-EGFP)chb2Tg). (A) Bright-field microscopic image of a zebrafish embryo 24 hpf. (B) The transgenic $\mathrm{Tg}$ (NC:NfsB-mCherry) zebrafish embryos express nuclear eGFP in the cardiomyocytes (green) and mCherry (red) in cells derived from the neural crest lineage at $36 \mathrm{hpf}$. (C-G) Colocalization of eGFP and mCherry from 26-30 hpf indicates the presence of cardiomyocytes of neural crest origin (yellow, arrows). (H-J) Dual-channel LSFM to capture the dynamic movement of atrioventricular (AV) valve leaflets from the transgenic Tg(fli1:GFP; $\mathrm{cmlc2}$ :mCherry) zebrafish embryos. An illustration of the AV valve leaflets in relation to the myocardium (cmlc:mCherry) and the endocardium (fli1:GFP) (H). AV valve leaflet closure (I) and opening (J) were captured at $100 \mathrm{fps}$. Myocardium (red) and endocardium (green) were concurrently acquired by the dual-channel imaging system. (K and $\mathbf{L}$ ) Cardiac architecture following doxorubicin treatment and $3-D$ rendering of the adult zebrafish heart. A crosssection through the atrium, ventricle, and bulbus arteriosus demonstrates the two leaflets of the AV valve (red) and of the ventriculo-bulbar (VB) valve (orange) (K). Throughout the duration of the study, control hearts exhibited a preserved architecture in comparison with doxorubicin-treated groups at days 3, 30, and 60 (L). (Reproduced with permission from ref. 58). Scale bars: $50 \mu \mathrm{m}$ (A-C); $10 \mu \mathrm{m}$ (insets of C-C on the right side); $200 \mu \mathrm{m}$ (I-L).

quantitation of cardiac volumes at days 3,30, and 60 after chemotherapy treatment (Figure 2L). Compared with control fish, doxorubicin-treatment fish had an acute decrease in myocardial and endocardial volumes at day 3, demonstrating global cardiac injury. Ventricular remodeling was notable at day 30, and by day 60 , the injured heart had completely regenerated and normal architecture was restored. The results demonstrate the suitability of LSFM combined with automated segmentation as a high-throughput method to monitor 3-D cardiac ultrastructural changes in adult zebrafish, with translational implications for drug discovery and modifiers of chemotherapy-induced cardiomyopathy.

\section{Three-dimensional tracking of cardiac regeneration in neonatal and adult mouse hearts}

The 3-D distribution of cardiac progenitor cells during both cardiac morphogenesis and regeneration remains poorly understood $(80,81)$. Two reporter systems were utilized along with LSFM imaging to identify cardiac progenitor lineages. A rainbow multicolor reporter system (82) was used to retrospectively identify the source of new cardiomyocytes in a mouse heart at P1 (Figure $3 \mathrm{~A}$ ) (83). In the $\alpha M H C^{\mathrm{Cre}} R 26^{\mathrm{VT2}}$ GK mouse model, Cre-mediated recombination of paired loxP sites resulted in expression of all four fluorescent proteins (cerulean, GFP, mOrange, and mCherry). Another fetal mouse model, Mesp $1^{\mathrm{Cre} /+}$ mice crossed with the Rosa2 $6^{t d T /+}$ reporter mice, allowed evaluation of the contribution of $\operatorname{tdT}^{+}$cells (yellow; hot) in an intact fetal mouse heart (Figure 3B). Embryos from timed mating of Mesp $1^{\text {Crel+ }}$ Rosa2 $6^{t d T /+}$ transgenic mice were isolated at E16.5, when the four-chambered heart had formed. Three-dimensional LSFM imaging showed the majority of the tdT-labeled cardiac cells arose from a Mesp1-expressing cell origin and allowed tracing of their 3-D distribution, proliferation, and tissue formation. Thus, light-sheet imaging allows for the elucidation of the organ-specific differentiation of stem cells in cardiac development and regeneration.

In addition, the relative ratio of ventricular volume to wall thickness provides insight into the regenerative capacity of cardiomyocyte proliferation at neonatal stages. A WT postnatal heart at P7 was imaged entirely by autofluorescence (Figure 3C) $(21,84)$. The 3-D reconstruction of the heart highlighted the ventricular cavity in yellow and rendered the myocardium by blue by a user-defined intensity threshold. Threedimensional, in toto imaging enables measurement of the thicknesses of the right ventricular wall $(\sim 300$ $\mu \mathrm{m})$, the septum $(\sim 650 \mu \mathrm{m})$, the left ventricular wall $(\sim 950 \mu \mathrm{m})$, and even ventricular volume in the intact mouse heart. Further, localization of ion channel distribution of in an adult heart is challenging to discern using conventional methods. With the use of LSFM, we revealed the spatial distribution of renal outer medullary potassium (ROMK) channels (green) after gene modulation and/or therapy in the intact heart (Figure 3D) $(21,85)$. An adult mouse heart of 7.5 months of age was imaged after tail vein injection of an adeno-associated virus vector 9 (AAV9) that employed a cardiac-specific troponin T promoter (cTnT) to drive cardiomyocyte gene expression of a customized construct in which the ROMK channel was fused at its C-terminus to one GFP molecule (AAV9-ROMK-GFP). Finally, this adult mouse heart was imaged in its entirety in 3-D to assess the cardiac-specific expression of an exogenous kidney potassium ion-channel that otherwise is not normally expressed in the heart.

\section{3-D calcific vasculopathy in the ApoE ${ }^{-/-}$mouse model of atherosclerosis}

Calcific vasculopathy is associated with increased mortality and morbidity, especially in patients with renal disease $(86,87)$. In addition to localizing the distribution of aortic calcium mineral, LSFM revealed the formation of calcification with high spatial resolution $(8 \mu \mathrm{m} \times 8 \mu \mathrm{m} \times 5 \mu \mathrm{m})$ in a 15-month-old $A p o E^{-/-}$female mouse that 

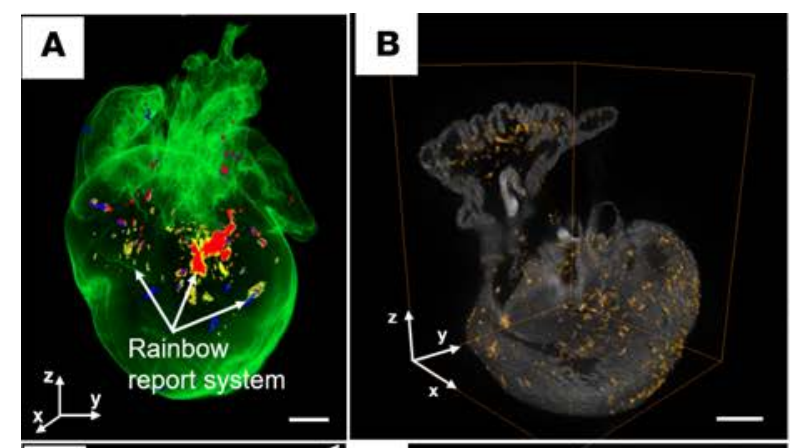

C
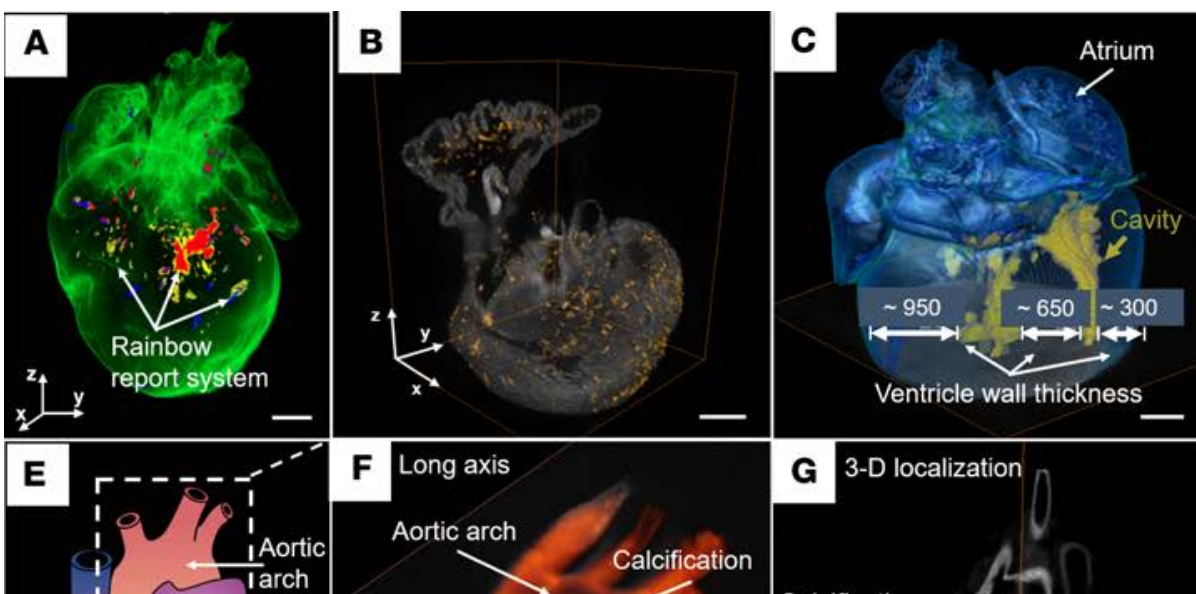

F Long axis

Aortic arch

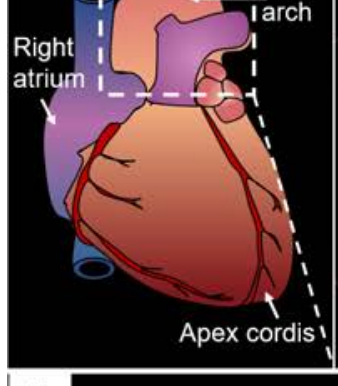

I
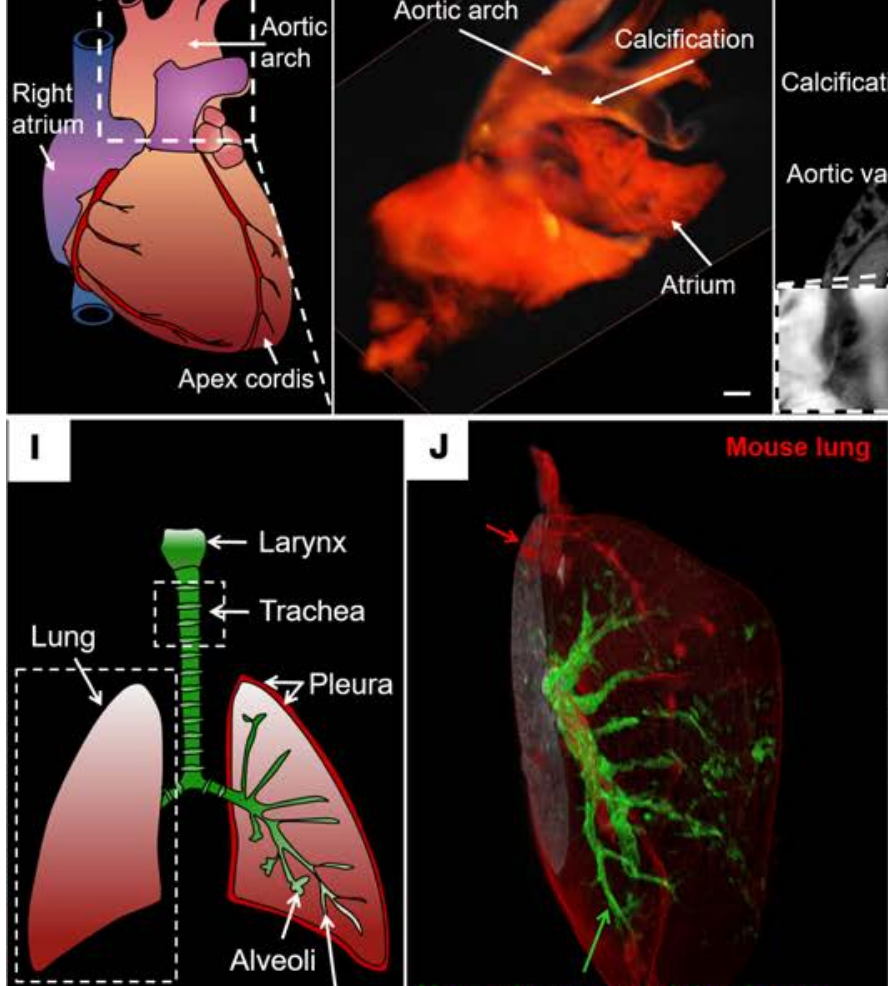

Calcification

Aortic valve
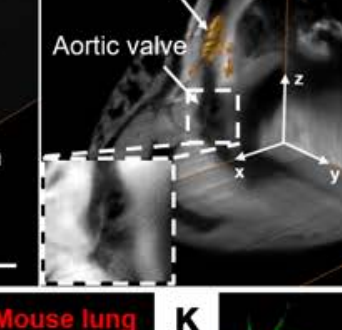

K
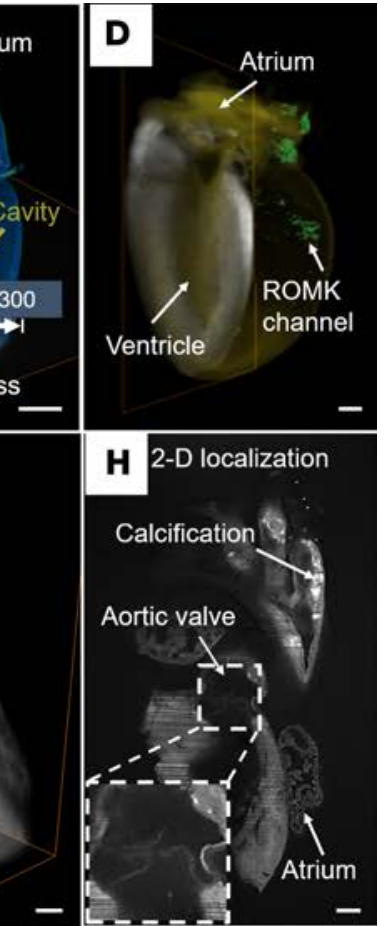

H 2-D localization

Calcification

Aortic valve
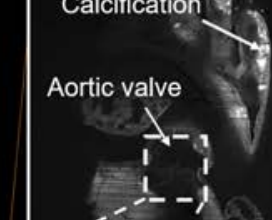

(1-1

$=---d^{\prime}$

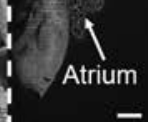

1. Atrium

\section{$-$}
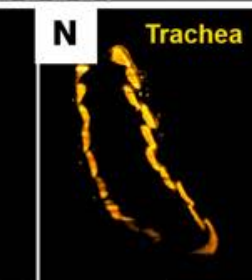

$\leftrightarrow$ Pleura
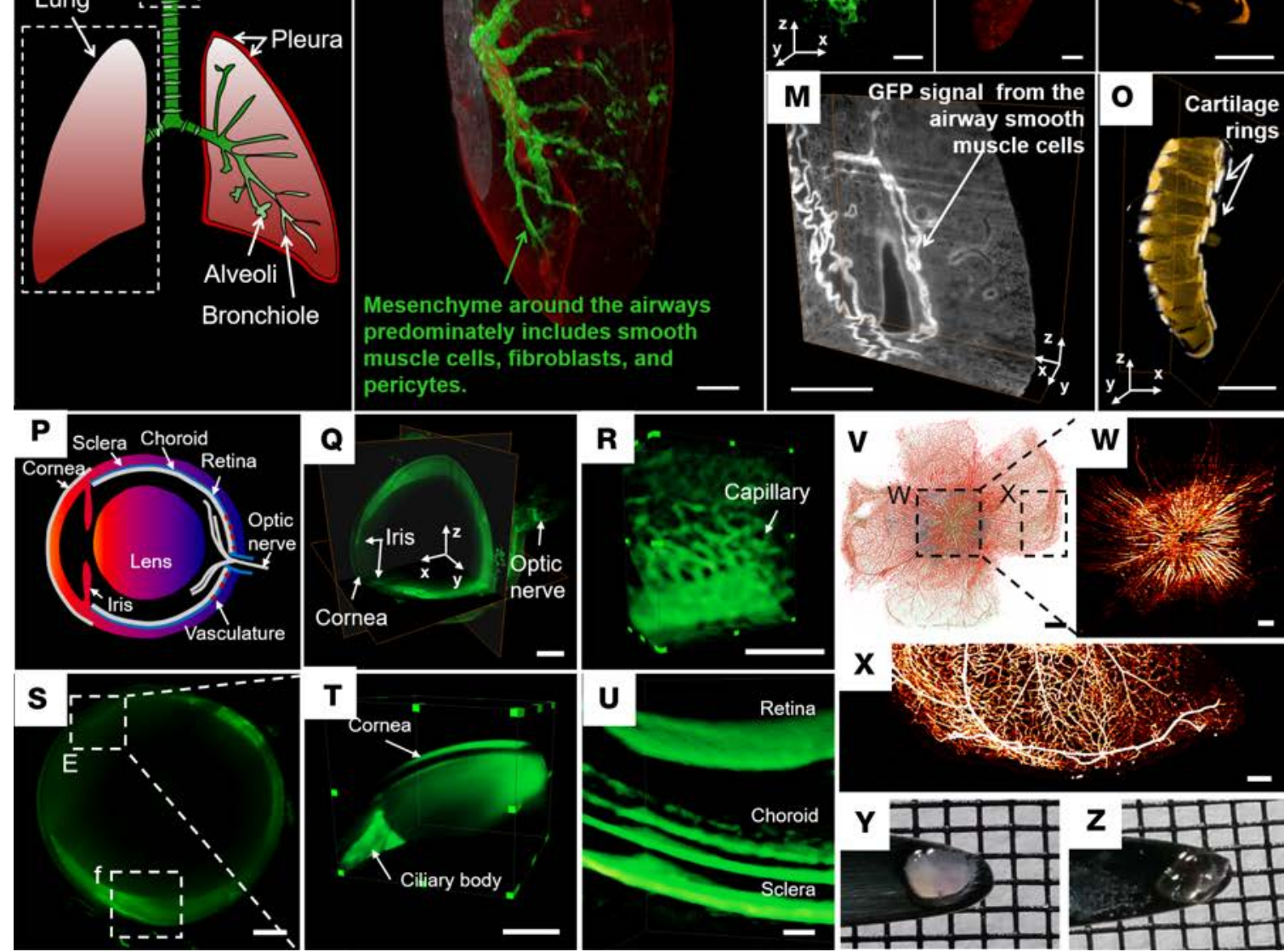

x

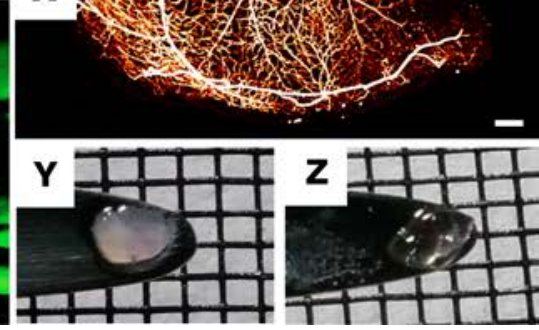


Figure 3. Three-dimensional interrogation of cardiovascular development and regeneration in neonatal and adult mouse models. (A and B) Cardiac progenitor lineage tracking in neonatal mouse hearts. Cre expression leads to cerulean, GFP, mOrange, and mCherry expression (A). Spatial distribution of $\mathrm{tdT}^{+}$cells (hot) in a neonatal heart from a Mesp $7^{\text {cre/+}}$ mouse crossed with the Rosa26 ${ }^{\mathrm{tdT} /+}$ reporter line (B). (C) The reconstructed heart reveals the small ventricular cavity (yellow) in a thick wall at P7. (D) Detection of GFP-tagged renal outer medullary potassium (ROMK) channel (green) in a 7.5-month-old adult mouse heart. (E-H) Calcific vasculopathy in a mouse atherosclerosis model. Illustration of cardiac anatomy delineating the areas imaged, including the heart base and aortic arch (E). Three-dimensional reconstruction shows calcium mineral (yellow) in a 15-month-old female apolipoprotein-deficient mouse (F). A 3-D orthogonal slice (G) and 2-D raw data (H) show distribution of aortic calcium mineral (brown and white, respectively). (I-O) Three-dimensional localization of developing mesenchyme from an 8-week-old reporter mouse lung. Cre expression (mGFP, green) was detected in lung mesenchymal cells, including airway, vascular smooth muscle cells, a variety of fibroblasts, vascular endothelial cells, and pericytes. Cre-negative cells expressed mTomato (red) (I-K). Two-dimensional (L) and 3-D (M) raw data of airway smooth muscle cells with high GFP. Tracheal architecture is visualized by the C-shaped rings (yellow) of hyaline cartilage ( $\mathbf{N}$ and $\mathbf{O}$ ). (P-Z) Label-free imaging of an intact albino mouse eye and fluorescence imaging of an rd10 eye using lightsheet microscopy. Illustration of a mouse eye includes the lens, cornea, sclera, choroid, retina, iris, and optic nerve (P). Light-sheet microscopy captured the 3-D orthogonal slice of the entire eyeball without changing the objective lens (Q). Three-dimensional vascular network in the posterior ocular system (R). Two-dimensional (S) and 3-D (T) structure of the cornea and ciliary body. Three-dimensional reconstruction of multilayer images reveals the retina, choroid, and sclera (U). The rd10 mouse is a model of autosomal recessive retinitis (V-X). Bipolar and ganglion cells express GFP (blue), while amacrine cells and vasculature are labeled with Alexa 594 (red) (V). Maximum intensity projection (MIP) images are presented in single-channel neurons expressing GFP (W) and single channel of vasculature labeled with Alexa 594 (X). Retina prior to (Y) and after (Z) simplified CLARITY are presented. Scale bars: 500 $\mu \mathrm{m}$ (A-D, Q, S-V); $1 \mathrm{~mm}$ (F-0); $200 \mu \mathrm{m}$ (R, W and $\mathbf{X}$ ). (Reproduced with permission from refs. 21, 83, 88.)

had been injected with a fluorescent bisphosphonate probe (5-FAM-ZOL; Biovinc) that binds calcium (Figure 3E) (88) and renders areas where this mineral is present fluorescent after injection. Three-dimensional reconstruction shows that calcification (yellow) is evident in the aortic arch (Figure 3F). Both the 3-D orthogonal slices (Figure $3 \mathrm{G}$ ) and 2-D raw data (Figure $3 \mathrm{H}$ ) unraveled the spatial location of aortic calcium mineral (brown in Figure $3 \mathrm{G}$ and white in Figure $3 \mathrm{H}$ ), and the aortic valve cusps were visible in the insets (Figure 3, $\mathrm{G}$ and $\mathrm{H}$ ). Thus, LSFM provides large-scale scanning of an adult mouse model of vascular calcification with translational implications for optimally controlling calcium and phosphate homeostasis in patients with chronic kidney disease (89) and for evaluating the efficacy of potential therapeutic agents.

\section{Visualization of developing pulmonary mesenchyme for lung morphogenesis}

In addition to the cardiovascular system, we have demonstrated that LSFM can be applied to investigate the developing pulmonary mesenchyme as a source of specification for lung development and function (Figure 3I) (90). An 8-week-old reporter mouse was generated by crossing the lung mesenchyme-specific Tbx4lung enhancer-driven Tet-On inducible Cre transgenic mouse (91) with a loxP-mTomato-STOP-loxP-mGFP (mTmG) fluorescent protein reporter mouse line (92). In this mouse, Cre-positive cells are green as a result of Cre-mediated floxed mTomato deletion, while Cre-negative cells are red as a result of mTomato expression (Figure 3J). The lung and trachea were harvested, perfused with $4 \%$ paraformaldehyde (PFA), and subjected to the simplified CLARITY protocol for tissue clearing $(21,85)$ after the mice were euthanized. Using light-sheet imaging, we demonstrated that doxycycline induction in the triple transgenic (Tbx4-rtTA TetO-Cre mT-mG) mouse line from E6.5-E18.5 resulted in mGFP expression only in lung mesenchymal cells, including airway and vascular smooth muscle cells, as well as a variety of fibroblasts, vascular endothelial cells, and pericytes. Using specific filtering thresholds, we were able to highlight an elevated GFP signal in airway smooth muscle cells; presumably, this intensity was due to the compacted nature of these cells as compared with low-intensity GFP from the scattered fibroblasts and pericytes (Figure 3K). The 2-D merged data (Figure 3L) and 3-D orthogonal slices (Figure $3 \mathrm{M}$ ) also allowed for tracing the developing mesenchyme with high spatial resolution at $2 \mu \mathrm{m} \times 2 \mu \mathrm{m} \times$ $1 \mu \mathrm{m}$. Furthermore, light-sheet imaging revealed the cross-section of C-shaped rings (yellow) of hyaline cartilage (Figure 3N) from a 3-D trachea (Figure 3O). While previous studies of lung development have focused on the lung epithelium, light-sheet imaging enables investigators to further investigate the lung mesenchyme as a critical source of inductive cues for a host of complex cell lineages during lung development and function.

\section{Label-free imaging of an intact mouse ocular system}

Ocular disorders are often early signs of cardiovascular disease $(93,94)$. While the existing imaging modalities - including fundus photography (95), confocal scanning laser ophthalmoscopy (96), and optical coherence tomography (97) - provide valuable 3-D ocular imaging, LSFM offers an advantage by enabling imaging of the entire globe with a single scan. This capacity for one-time scanning is achieved without the need to switch objective lenses when changing focus from anterior to posterior ocular structures due to the presence of the crystalline lens of the eye. An intact albino mouse was chosen for imaging because the 
Table 2. Overview of light-sheet techniques in comparison with other imaging modalities

\begin{tabular}{|c|c|c|c|c|}
\hline MRI/CT/US/PET/SPECT & $\begin{array}{l}\text { Versatile imaging modality } \\
\text { with high image contrast }\end{array}$ & $\begin{array}{c}\text { Anatomical, physiological, and } \\
\text { molecular imaging }\end{array}$ & Clinical and preclinical practice & $(146-151)$ \\
\hline PAT & $\begin{array}{l}\text { Optical illumination and } \\
\text { ultrasonic detection }\end{array}$ & $\begin{array}{l}\text { Absorption contrast and deep } \\
\text { penetration }\end{array}$ & $\begin{array}{l}\text { Vasculature, hemodynamics, } \\
\text { oxygen metabolism. }\end{array}$ & $(152,153)$ \\
\hline Confocal & $\begin{array}{l}\text { Spatial pinhole for optical } \\
\text { sectioning }\end{array}$ & $\begin{array}{l}\text { Elimination of out-of-focus } \\
\text { fluorescence }\end{array}$ & $\begin{array}{c}\text { Fluorescence imaging of cells and } \\
\text { tissues }\end{array}$ & $(154,155)$ \\
\hline MPM & Nonlinear optical imaging & Deep penetration and 3-D imaging & $\begin{array}{c}\text { From single-cell to behaving } \\
\text { organisms }\end{array}$ & $(156,157)$ \\
\hline STED/PALM/STORM & $\begin{array}{l}\text { Far-field subdiffraction limit } \\
\text { imaging }\end{array}$ & $\begin{array}{l}\text { Single molecular localization of live } \\
\text { ultrastructure }\end{array}$ & Molecular dynamics & $(158,159)$ \\
\hline
\end{tabular}

relative lack of melanin pigment rendered the ocular architecture intact and translucent, allowing for visualization of the cornea, conjunctiva, iris, sclera, choroid, retina, optic nerve, blood vessels, and lens without fluorescence staining (Figure 3, P-U). Endogenous autofluorescence served as the imaging contrast. LSFM has also been used to image an isolated retina from a mouse model of retinal degeneration ( $r d 10$ ) (Figure 3 , $\mathrm{V}-\mathrm{Z}$ ). In this model, the bipolar and ganglion cells in the retina expressed GFP (blue), while the amacrine cells and vasculature were labeled with Alexa 594 (red) (Figure 3V). The LSFM captured the neurons (Figure $3 \mathrm{~W}$ ) and vasculature (Figure $3 \mathrm{X}$ ) in the $r d 10$ model with a spatial resolution of $2 \mu \mathrm{m} \times 2 \mu \mathrm{m} \times 3 \mu \mathrm{m}$. In addition, we compared retinas with and without optical clearing (Figure 3, Y and Z). Light-sheet imaging was applied to image the zebrafish eye during development (98) and to quantify rodent retina development (99), further demonstrating the capability of LSFM for large-scale imaging of intact ocular systems.

Thus, in rodent models, LSFM enables the detailed analysis of developmental and vascular biology. In terms of cell lineage determinations, LSFM provides a powerful method to concurrently trace the 3-D distribution of cardiomyocytes that differentiate from numerous progenitors in an intact heart by taking advantage of the multichannel and large-scale capacities of the light-sheet imaging system. LSFM-generated images of organ-specific stem cell differentiation allow a more detailed understanding of cardiac development and regeneration. Furthermore, in comparison with CT or conventional optical microscopes (100-102), LSFM provides specific fluorescence from mineralized tissue and superb spatial resolution for large-scale scanning of vascular calcification in the $A p o E^{-/-}$mouse model of atherosclerosis, shedding light on translational optimization for regulating calcium and phosphate homeostasis in patients with chronic kidney disease (89). In addition, the demonstration of the role of developing pulmonary mesenchyme in mouse lung morphogenesis and the visualization of the entire mouse ocular system from the anterior to the posterior structures provide advances in optical imaging with great value to multiscale vascular biology and developmental cardiology.

\section{The future of LSFM}

Live imaging has transformed biomedical sciences by enabling visualization and analysis of dynamic cellular processes as they occur in their native context $(103,104)$. The advent of LSFM has led to widespread exploration of in vivo biological processes beyond the coverslip $(3,105)$. Currently, 4-D live imaging has made it possible to visualize biophysical and biochemical interactions in the freely moving embryos or rodents (106-109). The digital micromirror device-based light-field technique further enhances temporal resolution of LSFM for real-time volumetric imaging (110).

Among these optical methods, adaptive optics transforms conventional LSFM to compensate for optical aberrations and scattering by controlling the wavefronts (111-113). Furthermore, integration of selfreconstructing beams with LSFM generates a long and uniform light-sheet $(114,115)$, resulting in single cell imaging with two-photon Bessel beams (116). Further development of an optical lattice enables ultrathin light-sheet imaging from cellular to embryonic specimens (31). Meanwhile, another advance of LSFM is the ability to control light-sheet thickness by two electrically tunable lenses, adapting the position of lightsheet and light exposure independently throughout organisms within milliseconds (117). 
Parallel advances in pixel superresolution (118-120), deep learning (121-124), virtual reality (125128), optogenetics (129), and laser ablation (130) further provide complementary opportunities to elucidate cardiovascular architecture and function. A subvoxel LSFM is technically implemented for high-resolution, high-throughput volumetric imaging of cardiovascular development in a large field of view (22). This iterative resolution recovery method is transformative to improve inadequate focusing capability. For postimage processing, the development of a novel convolutional or recurrent neural network for automatic segmentation would likely bypass manual segmentation of large data sets. The study of interactive virtual reality demonstrates an efficient and robust framework for creating a user-directed microenvironment that can be used to uncover developmental cardiac mechanics and physiology with high spatiotemporal resolution.

\section{Conclusion}

In this review, we have summarized studies that have shown the capacity of LSFM for multiscale imaging to elucidate cardiopulmonary development, regeneration, and disease. Furthermore, we have shown that LSFM can provide detailed analysis of cardiovascular phenomena, ranging from embryonic heart development to calcific atherosclerotic disease in adult mice, and can be applied to samples, ranging from tissue clearing ex vivo to 4-D imaging in vivo. This framework builds on the high axial and temporal resolution for long-term, 3-D and 4-D visualization of in vivo cellular events, tissue morphogenesis, and organogenesis, with minimal photobleaching or phototoxicity. This strategy brings advanced imaging to studies of tissue injury, regeneration, and pathology, with multiscale applications to fundamental studies of cardiovascular development and translational work in cardiac anomalies and disease.

As compared with other optical imaging modalities, the unique feature of LSFM is the capacity to rapidly image the entire cardiovascular specimen within 30 seconds for zebrafish and 60 seconds for intact mouse hearts without the requiring image-column stitching. This methodology bypasses the need to move the tissue volume or light-sheet along the propagation of the illumination, thereby allowing for multiscale imaging for a wide range of specimens, from embryos to adults. This methodology further expands the field of view from hundreds of micrometers to tens of millimeters to cover the entire adult mouse heart with the spatiotemporal resolution needed to localize the progenitor cell fates. In addition to reducing the complexity of pre- and postprocessing of cardiac images, the optimized imaging strategy simplifies image acquisition and enhances the imaging system to provide dual-channel in vivo imaging at over $100 \mathrm{fps}$. The comparative advantages and applications among different imaging modalities are listed in Table 2 .

The limitations of LSFM for studying cardiovascular development and regeneration are the false-positive signals that result from the presence of pigment or residual hemoglobin in the ventricle. Numerous wavelengths of excitation and fluorophores with narrow emission spectra are required for distinguishing overlapping spectra artifacts from fluorescently labeled tissues. Other limitations that degrade image quality include photon absorption, scattering, and out-of-focus light, prone to the presence of stripes or shadow artifacts. Thus, optimization of optical clearing techniques (131-133) is critical to minimize the reduction or loss in fluorescence following prolonged optical clearing required for rodent models. Overall, we believe that LSFM will be transformative, as this multifunctional framework has potential to combine with new advances in optical imaging to provide great value for fundamental and translational research.

\section{Author contributions}

The authors would like to express gratitude to Dr. Lu Chen and Dr. Rongsong Li for preparation of this review. All of the authors contributed to the content in this review.

\section{Acknowledgments}

This study was supported by the NIH HL118650 (TKH), HL083015 (TKH), HL111437 (TKH), HL129727 (TKH), HL114709 (LLD, YT), and HL121019 (LLD, YT). JJH is supported by a T32 training grant from the NIH (HL007895) and an award from the UCLA Specialty Training and Advanced Research Program.

Address correspondence to: Tzung K. Hsiai, Department of Medicine (Cardiology) and Bioengineering, UCLA, 10833 Le Conte Avenue, CHS17-054A, Los Angeles, California 90095-1679, USA. Phone: 310.268.3839; Email: Thsiai@mednet.ucla.edu. 
1. Huisken J, Stainier DY. Selective plane illumination microscopy techniques in developmental biology. Development. 2009;136(12):1963-1975.

2. De Vos WH, et al. Invited review article: Advanced light microscopy for biological space research. Rev Sci Instrum. 2014;85(10):101101.

3. Power RM, Huisken J. A guide to light-sheet fluorescence microscopy for multiscale imaging. Nat Methods. 2017;14(4):360-373.

4. Bacallao R, Kiai K, Jesaitis L. Guiding Principles of Specimen Preservation for Confocal Fluorescence Microscopy. In: Pawley J, ed. Handbook of biological confocal microscopy. New York: Springer; 2006:368-380.

5. Miyaoka R, Lewellen T, Yu H, McDaniel D. Design of a depth of interaction (DOI) PET detector module. IEEE T Nucl Sci. 1998;45(3):1069-1073.

6. Tai YC, et al. MicroPET II: design, development and initial performance of an improved microPET scanner for small-animal imaging. Phys Med Biol. 2003;48(11):1519-1537.

7. Paxton R, Ambrose J. The EMI scanner. A brief review of the first 650 patients. Br J Radiol. 1974;47(561):530-565.

8. Prokop M. General principles of MDCT. Eur J Radiol. 2003;45 Suppl 1:S4-10.

9. Kelly KA, Allport JR, Tsourkas A, Shinde-Patil VR, Josephson L, Weissleder R. Detection of vascular adhesion molecule-1 expression using a novel multimodal nanoparticle. Circ Res. 2005;96(3):327-336.

10. van der Graaf M. In vivo magnetic resonance spectroscopy: basic methodology and clinical applications. Eur Biophys $J$. 2010;39(4):527-540

11. Ntziachristos V. Fluorescence molecular imaging. Annu Rev Biomed Eng. 2006;8:1-33.

12. Li C, et al. Proapoptotic protein Bim attenuates estrogen-enhanced survival in lymphangioleiomyomatosis. JCI Insight. 2016;1(19):e86629.

13. Patel MR, et al. ACCF proposed method for evaluating the appropriateness of cardiovascular imaging. J Am Coll Cardiol. 2005;46(8):1606-1613

14. Slavin GS, Bluemke DA. Spatial and temporal resolution in cardiovascular MR imaging: review and recommendations. Radiology. 2005;234(2):330-338.

15. Sanz J, Fayad ZA. Imaging of atherosclerotic cardiovascular disease. Nature. 2008;451(7181):953-957.

16. James ML, Gambhir SS. A molecular imaging primer: modalities, imaging agents, and applications. Physiol Rev. 2012;92(2):897-965.

17. Plana JC, et al. Expert consensus for multimodality imaging evaluation of adult patients during and after cancer therapy: a report from the American Society of Echocardiography and the European Association of Cardiovascular Imaging. Eur Heart $J$ Cardiovasc Imaging. 2014;15(10):1063-1093.

18. Fei P, et al. Cardiac Light-Sheet Fluorescent Microscopy for Multi-Scale and Rapid Imaging of Architecture and Function. Sci Rep. 2016;6:22489.

19. Guan Z, et al. Compact plane illumination plugin device to enable light sheet fluorescence imaging of multi-cellular organisms on an inverted wide-field microscope. Biomed Opt Express. 2016;7(1):194-208.

20. Lee J, et al. 4-Dimensional light-sheet microscopy to elucidate shear stress modulation of cardiac trabeculation. J Clin Invest. 2016;126(5):1679-1690.

21. Ding Y, et al. Light-sheet fluorescence imaging to localize cardiac lineage and protein distribution. Sci Rep. 2017;7:42209.

22. Fei P, et al. Sub-voxel light-sheet microscopy for high-resolution, high-throughput volumetric imaging of large biomedical specimens. bioRxiv. https://www.biorxiv.org/content/early/2018/04/18/255695. Published April 18, 2018. Accessed August 2, 2018.

23. Huisken J, Swoger J, Del Bene F, Wittbrodt J, Stelzer EH. Optical sectioning deep inside live embryos by selective plane illumination microscopy. Science. 2004;305(5686):1007-1009.

24. Scherz PJ, Huisken J, Sahai-Hernandez P, Stainier DY. High-speed imaging of developing heart valves reveals interplay of morphogenesis and function. Development. 2008;135(6):1179-1187.

25. Mickoleit M, et al. High-resolution reconstruction of the beating zebrafish heart. Nat Methods. 2014;11(9):919-922.

26. Chhetri RK, Amat F, Wan Y, Höckendorf B, Lemon WC, Keller PJ. Whole-animal functional and developmental imaging with isotropic spatial resolution. Nat Methods. 2015;12(12):1171-1178.

27. Dodt HU, et al. Ultramicroscopy: development and outlook. Neurophotonics. 2015;2(4):041407.

28. Sideris E, et al. Particle hydrogels based on hyaluronic acid building blocks. ACS Biomater Sci Eng. 2016;2(11):2034-2041.

29. Siedentopf H, Zsigmondy R. Uber sichtbarmachung und größenbestimmung ultramikoskopischer teilchen, mit besonderer anwendung auf goldrubingläser. Annalen der Physik. 1902;315(1):1-39.

30. Wu Y, et al. Spatially isotropic four-dimensional imaging with dual-view plane illumination microscopy. Nat Biotechnol. 2013;31(11):1032-1038.

31. Chen BC, et al. Lattice light-sheet microscopy: imaging molecules to embryos at high spatiotemporal resolution. Science. 2014;346(6208):1257998.

32. Keller PJ, Schmidt AD, Wittbrodt J, Stelzer EH. Reconstruction of zebrafish early embryonic development by scanned light sheet microscopy. Science. 2008;322(5904):1065-1069.

33. Alvers AL, Ryan S, Scherz PJ, Huisken J, Bagnat M. Single continuous lumen formation in the zebrafish gut is mediated by smoothened-dependent tissue remodeling. Development. 2014;141(5):1110-1119.

34. Truong TV, Supatto W, Koos DS, Choi JM, Fraser SE. Deep and fast live imaging with two-photon scanned light-sheet microscopy. Nat Methods. 2011;8(9):757-760.

35. Krzic U, Gunther S, Saunders TE, Streichan SJ, Hufnagel L. Multiview light-sheet microscope for rapid in toto imaging. Nat Methods. 2012;9(7):730-733.

36. Dodt HU, et al. Ultramicroscopy: three-dimensional visualization of neuronal networks in the whole mouse brain. Nat Methods. 2007;4(4):331-336.

37. Dodt HU, et al. Ultramicroscopy: three-dimensional visualization of neuronal networks in the whole mouse brain. Nat Methods. 2007;4(4):331-336.

38. Saghafi S, Becker K, Jährling N, Richter M, Kramer ER, Dodt HU. Image enhancement in ultramicroscopy by improved laser light sheets. J Biophotonics. 2010;3(10-11):686-695. 
39. Saghafi S, Becker K, Hahn C, Dodt HU. 3D-ultramicroscopy utilizing aspheric optics. J Biophotonics. 2014;7(1-2):117-125.

40. Voie AH, Burns DH, Spelman FA. Orthogonal-plane fluorescence optical sectioning: three-dimensional imaging of macroscopic biological specimens. J Microsc. 1993;170(Pt 3):229-236.

41. Voie AH, Spelman FA. Three-dimensional reconstruction of the cochlea from two-dimensional images of optical sections. Comput Med Imaging Graph. 1995;19(5):377-384.

42. Voie AH. Imaging the intact guinea pig tympanic bulla by orthogonal-plane fluorescence optical sectioning microscopy. Hear Res. 2002;171(1-2):119-128.

43. Buytaert JA, Dirckx JJ. Design and quantitative resolution measurements of an optical virtual sectioning three-dimensional imaging technique for biomedical specimens, featuring two-micrometer slicing resolution. J Biomed Opt. 2007;12(1):014039.

44. Richardson DS, Lichtman JW. Clarifying Tissue Clearing. Cell. 2015;162(2):246-257.

45. Richardson DS, Lichtman JW. SnapShot: Tissue Clearing. Cell. 2017;171(2):496-496.e1.

46. Fehrenbach J, Weiss P, Lorenzo C. Variational algorithms to remove stationary noise: applications to microscopy imaging. IEEE Trans Image Process. 2012;21(10):4420-4430.

47. Fehrenbach J, Weiss P. Processing stationary noise: Model and parameter selection in variational methods. Cornell University Library. https://arxiv.org/abs/1307.4592. Accessed August 2, 2018.

48. Liang X, et al. Stripe artifact elimination based on nonsubsampled contourlet transform for light sheet fluorescence microscopy. J Biomed Opt. 2016;21(10):106005.

49. Buytaert JA, Dirckx JJ. Tomographic imaging of macroscopic biomedical objects in high resolution and three dimensions using orthogonal-plane fluorescence optical sectioning. Appl Opt. 2009;48(5):941-948.

50. Santi PA, Johnson SB, Hillenbrand M, GrandPre PZ, Glass TJ, Leger JR. Thin-sheet laser imaging microscopy for optical sectioning of thick tissues. BioTechniques. 2009;46(4):287-294.

51. Buytaert JA, Descamps E, Adriaens D, Dirckx JJ. The OPFOS Microscopy Family: High-Resolution Optical Sectioning of Biomedical Specimens. Anat Res Int. 2012;2012:206238.

52. Huisken J, Stainier DY. Even fluorescence excitation by multidirectional selective plane illumination microscopy (mSPIM). Opt Lett. 2007;32(17):2608-2610.

53. Mertz J, Kim J. Scanning light-sheet microscopy in the whole mouse brain with HiLo background rejection. J Biomed Opt. 2010;15(1):016027.

54. Swoger J, Verveer P, Greger K, Huisken J, Stelzer EH. Multi-view image fusion improves resolution in three-dimensional microscopy. Opt Express. 2007;15(13):8029-8042.

55. Preibisch S, Saalfeld S, Schindelin J, Tomancak P. Software for bead-based registration of selective plane illumination microscopy data. Nat Methods. 2010;7(6):418-419.

56. Weber M, Huisken J. Light sheet microscopy for real-time developmental biology. Curr Opin Genet Dev. 2011;21(5):566-572.

57. Tomer R, Khairy K, Amat F, Keller PJ. Quantitative high-speed imaging of entire developing embryos with simultaneous multiview light-sheet microscopy. Nat Methods. 2012;9(7):755-763.

58. Packard RRS, et al. Automated Segmentation of Light-Sheet Fluorescent Imaging to Characterize Experimental DoxorubicinInduced Cardiac Injury and Repair. Sci Rep. 2017;7(1):8603.

59. Baek KI, et al. Advanced microscopy to elucidate cardiovascular injury and regeneration: 4D light-sheet imaging [published online ahead of print May 9, 2018]. Progr Biophys Mol Biol. https://doi.org/10.1016/j.pbiomolbio.2018.05.003.

60. Li YX, Zdanowicz M, Young L, Kumiski D, Leatherbury L, Kirby ML. Cardiac neural crest in zebrafish embryos contributes to myocardial cell lineage and early heart function. Dev Dyn. 2003;226(3):540-550.

61. Cavanaugh AM, Huang J, Chen JN. Two developmentally distinct populations of neural crest cells contribute to the zebrafish heart. Dev Biol. 2015;404(2):103-112.

62. Hatzistergos KE, et al. Transient Bone Morphogenic Protein Antagonism Directs Differentiation of Ipscs Into the Cardiac Neural Crest Ckit+ Myocardial Progenitor Lineages. Circulation. 2018;132(Suppl_3):A19282

63. Hatzistergos KE, et al. cKit+ cardiac progenitors of neural crest origin. Proc Natl Acad Sci USA. 2015;112(42):13051-13056.

64. Yalcin HC, Shekhar A, McQuinn TC, Butcher JT. Hemodynamic patterning of the avian atrioventricular valve. Dev Dyn. 2011;240(1):23-35.

65. Beis D, et al. Genetic and cellular analyses of zebrafish atrioventricular cushion and valve development. Development 2005;132(18):4193-4204.

66. Beis D, Stainier DY. In vivo cell biology: following the zebrafish trend. Trends Cell Biol. 2006;16(2):105-112.

67. Lee J, et al. Moving domain computational fluid dynamics to interface with an embryonic model of cardiac morphogenesis PLoS One. 2013;8(8):e72924

68. Ding Y, et al. Light-Sheet Imaging to Elucidate Cardiovascular Injury and Repair. Curr Cardiol Rep. 2018;20(5):35.

69. de Pater E, et al. Distinct phases of cardiomyocyte differentiation regulate growth of the zebrafish heart. Development. 2009;136(10):1633-1641.

70. Lazic S, Scott IC. Mef2cb regulates late myocardial cell addition from a second heart field-like population of progenitors in zebrafish. Dev Biol. 2011;354(1):123-133.

71. Zhou Y, et al. Latent TGF- $\beta$ binding protein 3 identifies a second heart field in zebrafish. Nature. 2011;474(7353):645-648.

72. Staudt DW, Liu J, Thorn KS, Stuurman N, Liebling M, Stainier DY. High-resolution imaging of cardiomyocyte behavior reveals two distinct steps in ventricular trabeculation. Development. 2014;141(3):585-593.

73. Paige SL, Plonowska K, Xu A, Wu SM. Molecular regulation of cardiomyocyte differentiation. Circ Res. 2015;116(2):341-353.

74. Jiménez-Amilburu V, et al. In Vivo Visualization of Cardiomyocyte Apicobasal Polarity Reveals Epithelial to Mesenchymal-like Transition during Cardiac Trabeculation. Cell Rep. 2016;17(10):2687-2699.

75. Lu F, Langenbacher AD, Chen JN. Transcriptional Regulation of Heart Development in Zebrafish. J Cardiovasc Dev Dis. 2016;3(2):14.

76. Ding Y, et al. Observation of mesenteric microcirculatory disturbance in rat by laser oblique scanning optical microscopy. Sci Rep. 2013;3:1762.

77. Lee J, et al. A Rapid Capillary-Pressure Driven Micro-Channel to Demonstrate Newtonian Fluid Behavior of Zebrafish Blood 
at High Shear Rates. Sci Rep. 2017;7(1):1980.

78. Lee J, et al. Hemodynamics and ventricular function in a zebrafish model of injury and repair. Zebrafish. 2014;11(5):447-454.

79. Ding Y, et al. Haploinsufficiency of target of rapamycin attenuates cardiomyopathies in adult zebrafish. Circ Res. 2011;109(6):658-669.

80. Bergmann O, et al. Evidence for cardiomyocyte renewal in humans. Science. 2009;324(5923):98-102.

81. Hsieh PC, et al. Evidence from a genetic fate-mapping study that stem cells refresh adult mammalian cardiomyocytes after injury. Nat Med. 2007;13(8):970-974.

82. Rinkevich Y, Lindau P, Ueno H, Longaker MT, Weissman IL. Germ-layer and lineage-restricted stem/progenitors regenerate the mouse digit tip. Nature. 2011;476(7361):409-413.

83. Sereti KI, et al. Analysis of cardiomyocyte clonal expansion during mouse heart development and injury. Nat Commun. 2018;9(1):754.

84. Yokota T, et al. p38 MAP Kinase Regulates Chamber Specific Postnatal Remodelling of Cardiac Ventricles. Circ Res. 2016;119(Suppl 1):A68.

85. Sung K, et al. Simplified three-dimensional tissue clearing and incorporation of colorimetric phenotyping. Sci Rep 2016;6:30736.

86. Rosito GA, et al. Pericardial fat, visceral abdominal fat, cardiovascular disease risk factors, and vascular calcification in a community-based sample: the Framingham Heart Study. Circulation. 2008;117(5):605-613.

87. Mizobuchi M, Towler D, Slatopolsky E. Vascular calcification: the killer of patients with chronic kidney disease. J Am Soc Nephrol. 2009;20(7):1453-1464.

88. Hsu JJ, et al. Effects of teriparatide on morphology of aortic calcification in aged hyperlipidemic mice. Am J Physiol Heart Circ Physiol. 2018;314(6):H1203-H1213.

89. Shanahan CM, Crouthamel MH, Kapustin A, Giachelli CM. Arterial calcification in chronic kidney disease: key roles for calcium and phosphate. Circ Res. 2011;109(6):697-711.

90. McCulley D, Wienhold M, Sun X. The pulmonary mesenchyme directs lung development. Curr Opin Genet Dev. 2015;32:98-105.

91. Zhang W, et al. Spatial-temporal targeting of lung-specific mesenchyme by a Tbx4 enhancer. BMC Biol. 2013;11:111.

92. Muzumdar MD, Tasic B, Miyamichi K, Li L, Luo L. A global double-fluorescent Cre reporter mouse. Genesis. 2007;45(9):593-605.

93. Goldzweig CL, Rowe S, Wenger NS, MacLean CH, Shekelle PG. Preventing and managing visual disability in primary care: clinical applications. JAMA. 2004;291(12):1497-1502.

94. Rowe S, MacLean $\mathrm{CH}$, Shekelle PG. Preventing visual loss from chronic eye disease in primary care: scientific review. JAMA. 2004;291(12):1487-1495.

95. Moss SE, Klein R, Kessler SD, Richie KA. Comparison between ophthalmoscopy and fundus photography in determining severity of diabetic retinopathy. Ophthalmology. 1985;92(1):62-67.

96. Seeliger MW, et al. In vivo confocal imaging of the retina in animal models using scanning laser ophthalmoscopy. Vision Res. 2005;45(28):3512-3519.

97. Drexler W, Morgner U, Ghanta RK, Kärtner FX, Schuman JS, Fujimoto JG. Ultrahigh-resolution ophthalmic optical coherence tomography. Nat Med. 2001;7(4):502-507.

98. Icha J, Schmied C, Sidhaye J, Tomancak P, Preibisch S, Norden C. Using light sheet fluorescence microscopy to image zebrafish eye development. J Vis Exp. 2016;(110):e53966.

99. Singh JN, Nowlin TM, Seedorf GJ, Abman SH, Shepherd DP. Quantifying three-dimensional rodent retina vascular development using optical tissue clearing and light-sheet microscopy. J Biomed Opt. 2017;22(7):76011.

100. Ding Y, et al. Laser oblique scanning optical microscopy (LOSOM) for phase relief imaging. Opt Express. 2012;20(13):14100-14108.

101. Lu Y, et al. An integrated quad-modality molecular imaging system for small animals. J Nucl Med. 2014;55(8):1375-1379.

102. Ding Y, et al. In vivo study of endometriosis in mice by photoacoustic microscopy. J Biophotonics. 2015;8(1-2):94-101.

103. Amos B. Lessons from the history of light microscopy. Nat Cell Biol. 2000;2(8):E151-E152.

104. Yuste R. Fluorescence microscopy today. Nat Methods. 2005;2(12):902-904.

105. Glaser AK, et al. Light-sheet microscopy for slide-free non-destructive pathology of large clinical specimens. Nat Biomed Eng. 2017;1(7):0084.

106. Prevedel R, et al. Simultaneous whole-animal 3D imaging of neuronal activity using light-field microscopy. Nat Methods. 2014;11(7):727-730

107. McGorty R, Liu H, Kamiyama D, Dong Z, Guo S, Huang B. Open-top selective plane illumination microscope for conventionally mounted specimens. Opt Express. 2015;23(12):16142-16153.

108. Cong L, et al. Rapid whole brain imaging of neural activity in freely behaving larval zebrafish (Danio rerio). Elife. 2017;6:e28158.

109. Skocek O, et al. High-speed volumetric imaging of neuronal activity in freely moving rodents. Nat Methods. 2018;15(6):429-432.

110. Wolff JM, Castro D, Arbeláez P, Forero-Shelton M. Light-sheet enhanced resolution of light field microscopy for rapid imaging of large volumes. SPIE BiOS. 2018;10499:104991U.

111. Masson A, Escande P, Frongia C, Clouvel G, Ducommun B, Lorenzo C. High-resolution in-depth imaging of optically cleared thick samples using an adaptive SPIM. Sci Rep. 2015;5:16898.

112. Wilding D, Pozzi P, Soloviev O, Vdovin G, Verhaegen M. Adaptive illumination based on direct wavefront sensing in a lightsheet fluorescence microscope. Opt Express. 2016;24(22):24896-24906.

113. Liu TL, et al. Observing the cell in its native state: Imaging subcellular dynamics in multicellular organisms. Science. 2018;360(6386):eaaq1392.

114. Fahrbach FO, Rohrbach A. A line scanned light-sheet microscope with phase shaped self-reconstructing beams. Opt Express. 2010;18(23):24229-24244.

115. Fahrbach FO, Gurchenkov V, Alessandri K, Nassoy P, Rohrbach A. Self-reconstructing sectioned Bessel beams offer submicron optical sectioning for large fields of view in light-sheet microscopy. Opt Express. 2013;21(9):11425-11440.

116. Planchon TA, et al. Rapid three-dimensional isotropic imaging of living cells using Bessel beam plane illumination. Nat Meth ods. 2011;8(5):417-423. 
117. Chmielewski AK, et al. Fast imaging of live organisms with sculpted light sheets. Sci Rep. 2015;5:9385.

118. Zheng G, Lee SA, Yang S, Yang C. Sub-pixel resolving optofluidic microscope for on-chip cell imaging. Lab Chip. 2010;10(22):3125-3129.

119. Zheng G, Lee SA, Antebi Y, Elowitz MB, Yang C. The ePetri dish, an on-chip cell imaging platform based on subpixel perspective sweeping microscopy (SPSM). Proc Natl Acad Sci USA. 2011;108(41):16889-16894.

120. Greenbaum A, Sikora U, Ozcan A. Field-portable wide-field microscopy of dense samples using multi-height pixel super-resolution based lensfree imaging. Lab Chip. 2012;12(7):1242-1245.

121. Lawrence S, Giles CL, Tsoi AC, Back AD. Face recognition: a convolutional neural-network approach. IEEE Trans Neural Netw. 1997;8(1):98-113.

122.Krizhevsky A, Sutskever I, Hinton GE. ImageNet Classification with Deep Convolutional Neural Networks. In: Neural Information Processing Systems Conference; December 3-8, 2012; Lake Tahoe, NV.

123. Long J, Shelhamer E, Darrell T. Fully Convolutional Networks for Semantic Segmentation. In: The IEEE Conference on Computer Vision and Pattern Recognition (CVPR); June 7-12, 2015; Boston, MA; 3431-3440.

124. Zheng S, et al. Conditional Random Fields as Recurrent Neural Networks. In: The IEEE International Conference on Computer Vision (ICCV); December 11-18, 2015; Santiago, Chile; 1529-1537.

125. Peng H, Ruan Z, Long F, Simpson JH, Myers EW. V3D enables real-time 3D visualization and quantitative analysis of largescale biological image data sets. Nat Biotechnol. 2010;28(4):348-353.

126. Eliceiri KW, et al. Biological imaging software tools. Nat Methods. 2012;9(7):697-710.

127. Peng $\mathrm{H}$, et al. Virtual finger boosts three-dimensional imaging and microsurgery as well as terabyte volume image visualization and analysis. Nat Commun. 2014;5:4342.

128. Ding Y, et al. Integrating light-sheet imaging with virtual reality to recapitulate developmental cardiac mechanics. JCI Insight. 2017;2(22):e97180.

129. Arrenberg AB, Stainier DY, Baier H, Huisken J. Optogenetic control of cardiac function. Science. 2010;330(6006):971-974.

130. Engelbrecht CJ, Greger K, Reynaud EG, Krzic U, Colombelli J, Stelzer EH. Three-dimensional laser microsurgery in light-sheet based microscopy (SPIM). Opt Express. 2007;15(10):6420-6430.

131. Murray E, et al. Simple, Scalable Proteomic Imaging for High-Dimensional Profiling of Intact Systems. Cell. 2015;163(6):1500-1514.

132. Neckel PH, Mattheus U, Hirt B, Just L, Mack AF. Large-scale tissue clearing (PACT): Technical evaluation and new perspectives in immunofluorescence, histology, and ultrastructure. Sci Rep. 2016;6:34331.

133. Cuccarese MF, et al. Heterogeneity of macrophage infiltration and therapeutic response in lung carcinoma revealed by $3 \mathrm{D}$ organ imaging. Nat Commun. 2017;8:14293.

134.Fuchs E, Jaffe J, Long R, Azam F. Thin laser light sheet microscope for microbial oceanography. Opt Express. 2002;10(2):145-154

135. Holekamp TF, Turaga D, Holy TE. Fast three-dimensional fluorescence imaging of activity in neural populations by objectivecoupled planar illumination microscopy. Neuron. 2008;57(5):661-672.

136. Galland R, Grenci G, Aravind A, Viasnoff V, Studer V, Sibarita JB. 3D high- and super-resolution imaging using single-objective SPIM. Nat Methods. 2015;12(7):641-644.

137. Dunsby C. Optically sectioned imaging by oblique plane microscopy. Opt Express. 2008;16(25):20306-20316.

138. Tokunaga M, Imamoto N, Sakata-Sogawa K. Highly inclined thin illumination enables clear single-molecule imaging in cells. Nat Methods. 2008;5(2):159-161.

139. Bouchard MB, et al. Swept confocally-aligned planar excitation (SCAPE) microscopy for high speed volumetric imaging of behaving organisms. Nat Photonics. 2015;9(2):113-119.

140. Amat F, et al. Fast, accurate reconstruction of cell lineages from large-scale fluorescence microscopy data. Nat Methods. 2014;11(9):951-958.

141. Kumar A, et al. Dual-view plane illumination microscopy for rapid and spatially isotropic imaging. Nat Protoc. 2014;9(11):2555-2573.

142. Wu Y, et al. Simultaneous multiview capture and fusion improves spatial resolution in wide-field and light-sheet microscopy. Optica. 2016;3(8):897-910.

143. Gao L, et al. Noninvasive imaging beyond the diffraction limit of 3D dynamics in thickly fluorescent specimens. Cell. 2012;151(6):1370-1385.

144. Welf ES, et al. Quantitative Multiscale Cell Imaging in Controlled 3D Microenvironments. Dev Cell. 2016;36(4):462-475.

145. Vettenburg T, et al. Light-sheet microscopy using an Airy beam. Nat Methods. 2014;11(5):541-544.

146. Kalender WA. X-ray computed tomography. Phys Med Biol. 2006;51(13):R29-R43.

147. Quon A, Gambhir SS. FDG-PET and beyond: molecular breast cancer imaging. J Clin Oncol. 2005;23(8):1664-1673.

148. Lauterbur PC. Image Formation by Induced Local Interactions: Examples Employing Nuclear Magnetic Resonance. Nature. 1973;242(244):190.

149. Mariani G, et al. A review on the clinical uses of SPECT/CT. Eur J Nucl Med Mol Imaging. 2010;37(10):1959-1985.

150. Fenster A, Downey DB, Cardinal HN. Three-dimensional ultrasound imaging. Phys Med Biol. 2001;46(5):R67-R99.

151. Wagner A, et al. Contrast-enhanced MRI and routine single photon emission computed tomography (SPECT) perfusion imaging for detection of subendocardial myocardial infarcts: an imaging study. Lancet. 2003;361(9355):374-379.

152. Wang LV, Hu S. Photoacoustic tomography: in vivo imaging from organelles to organs. Science. 2012;335(6075):1458-1462.

153. Beard P. Biomedical photoacoustic imaging. Interface Focus. 2011;1(4):602-631.

154. Marvin M, inventor; Marvin M, assignee. Microscopy apparatus. US grant US3013467A. November 7, 1957.

155. Inoué S. Foundations of Confocal Scanned Imaging in Light Microscopy. In: Pawley JB, ed. Handbook of biological confocal microscopy. New York, NY: Springer; 2006:1-19.

156. Denk W, Strickler JH, Webb WW. Two-photon laser scanning fluorescence microscopy. Science. 1990;248(4951):73-76.

157. Zipfel WR, Williams RM, Webb WW. Nonlinear magic: multiphoton microscopy in the biosciences. Nat Biotechnol. 2003;21(11):1369-1377.

158. Hell SW. Microscopy and its focal switch. Nat Methods. 2009;6(1):24-32.

159. Huang B, Bates M, Zhuang X. Super-resolution fluorescence microscopy. Annu Rev Biochem. 2009;78:993-1016. 